\title{
Stimulus affective valence reverses spatial compatibility effect
}

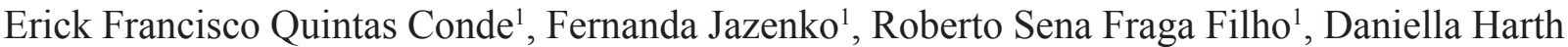 \\ da Costa $^{1}$, Nelson Torro-Alves ${ }^{2}$, Mikael Cavallet ${ }^{3}$ and Luiz G Gawryszewski ${ }^{1}$ \\ 1 - Universidade Federal Fluminense, Niterói, RJ, Brazil \\ 2 - Universidade Federal da Paraíba, João Pessoa, PB, Brazil \\ 3 - Universidade de São Paulo, São Paulo, SP, Brazil
}

\begin{abstract}
In spatial compatibility tasks, the Reaction Time to right-side stimuli is shorter for right key responses (compatible condition) than for left key responses (incompatible condition) and vice-versa for left-side stimuli. Similar results have been found when the stimulus location is not relevant for response selection, such as in the Simon task. The Simon effect is the difference between the reaction times for non-corresponding and corresponding conditions. The Simon effect and its variants may be modulated by using emotional stimuli. However, until now, no work has studied how the affective valence of a stimulus influences spatial compatibility effects along the horizontal dimension. The present study investigated this issue by using small lateralized figures of soccer team players as stimuli. In the experiment, a compatible or incompatible response was chosen according to the team shirt. In one block, for the Favorite team, the volunteers had to press the key on the same side as the stimulus hemifield but the opposite-side key for the Rival team. In the other block, a reverse code had to be used. Fourteen right-handed volunteers were tested. Mean reaction times were subjected to analysis of variance with the following variables: Preference (Favorite/Rival), Hemifield (Left/Right), and Response Key (Left/Right). A three-way interaction was found $\left(F_{1,13}=6.60, p=.023\right)$, showing that the spatial compatibility effects depended on Preference. The Favorite team player elicited the usual spatial compatibility pattern, but for the Rival team player, the reverse effect was found, with incompatible responses being faster than compatible responses. We propose that this modulation may result from approach/avoidance reactions to the Favorite and Rival teams, respectively. Moreover, we suggest as a corollary that the classic spatial compatibility task is a powerful tool for investigating approach/ avoidance effects. Keywords: affective valence, spatial compatibility, approach/avoidance, emotion, motor control, attention
\end{abstract}

Received 8 February 2011; received in revised form 31 March 2011; accepted 4 April 2011. Available on line 15 June 2011

\section{Introduction}

The role of emotion in decision making has been intensively studied in recent years (Damasio, 1994, 2010; Tamietto \& de Gelder, 2010) and involves both conscious and unconscious mechanisms (for review, see Tamietto \& de Gelder, 2010). In this paper, we show that the affective valence of a stimulus may modulate the spatial compatibility effects that arise from the

Erick Francisco Quintas Conde, Fernanda Jazenko, Roberto Sena Fraga Filho, Daniella Harth da Costa, and Luiz G Gawryszewski, Universidade Federal Fluminense, Departamento de Neurobiologia, ProgramadePós-graduaçãoemNeurociências, Brazil.Nelson TorroAlves, Universidade Federal da Paraíba, CCHLA, Departamento de Psicologia, Brazil. Mikael Cavallet, Universidade de São Paulo, Departamento de Psicologia, Faculdade de Filosofia, Ciências e Letras de Ribeirão Preto, Brazil. Correspondence regarding this article should be directed to: Luiz Gawryszewski, Departamento de Neurobiologia, Universidade Federal Fluminense, Centro de Estudos Gerais, Instituto de Biologia, Caixa Postal 100180, CEP 24020-971, Niterói, RJ, Brazil. Phone: +55-21-2629-2271. E-mail: gawryszewski_lg@yahoo.com.br relationship between the locations of the stimuli and response keys.

Spatial compatibility has a strong effect on Manual Reaction Time (MRT) to visual stimuli (Anzola, Bertoloni, Buchtel, \& Rizzolatti, 1977). When the side of a stimulus coincides with the side of the key (compatible condition), the MRT is approximately 50 ms shorter than when the stimulus and key are located on opposite sides (incompatible condition). The now classic paper of Anzola et al. (1977) showed that this effect occurs in a choice spatial task but not in a simple MRT task and is attributable to the spatial relationship between the locations of the stimulus and key, not to the location of the responding hand. Although the simple MRT task shows differences of approximately 2-3 ms that can be related to the interhemispheric conduction time (Poffenberger, 1912; Berlucchi, Crea, Di Stefano, \& Tassinari, 1977), the effects of spatial compatibility are at least 10 -fold greater. The spatial compatibility effect (of approximately $50 \mathrm{~ms}$ ) has been extensively studied in the last three decades. It occurs along both the horizontal and vertical meridians and depends on 
the relative positions of the stimuli and keys, not on the absolute positions. The spatial compatibility code is relative to the stimulus position and location of the hand action, not the hand side (Anzola et al., 1977; Gawryszewski et al., 2008; Pellicano et al., 2010; Proctor \& Vu, 2006; Riggio, Gawryszewski, \& Umiltà, 1986; Umiltà \& Nicoletti, 1990).

The Simon effect is another effect generated by the spatial relationship between the stimulus and key positions. However, in the Simon task, the response selection is based on nonspatial stimulus features, such as form and color. For example, when a lateralized red stimulus appears, the participant is instructed to press the left key, but when the stimulus is blue, the participant must press the right key or vice-versa. The left and right stimulus location varies randomly among trials. Although the spatial location of the stimulus is an irrelevant feature for the response selection, when the sides of the stimulus and key are the same (corresponding condition), the MRT is shorter (approximately 20-30 ms) than when the stimulus and key are located on opposite sides (for review, see Lu \& Proctor, 1995; Proctor \& Vu, 2006; Pellicano et al., 2010; Rubichi \& Nicoleti, 2006). Notably, the Simon effect results from the implicit processing of the relationship between the stimulus and key locations, and its magnitude is approximately half of the spatial compatibility effect, in which the location must be explicitly processed before the response is emitted.

Stimulus valence is linked with avoidance and approach behavior (for review, see Alves, Fukusima, \& Aznar-Casanova, 2008; Markman \& Brendl, 2005; Proctor \& Zhang, 2010). For example, people are faster at responding with approach for positive stimuli and avoidance for negative stimuli compared with the opposite mapping (Chen \& Bargh, 1999; Zhang \& Proctor, 2008; De Houwer, Crombez, Baeyens, \& Hermans, 2001). Chen and Bargh (1999) found that pulling movements were faster than pushing movements for positive words and the reverse for negative words (i.e., pushing movements were faster than pulling movements) when participants were requested to evaluate negative and positive words by moving a lever away from the body (avoidance) or toward the body (approach). The authors suggested that positive evaluations (positive stimuli) produce approach tendencies, whereas negative evaluations (negative stimuli) produce avoidance tendencies. Müsseler, Aschersleben, Arning and Proctor (2009) examined the effects of spatial stimulus-response compatibility in natural scenes. In a short video viewed from a driver's perspective, a pedestrian entered the street from the left or right side. Participants were required to turn toward a person calling a taxi and away from a person who carelessly entered the street. They observed a reverse compatibility effect in dangerous situations in natural scenes (a person carelessly walking into the road). More specifically, they found that spatially incompatible responses (i.e., turn away from stimuli of negative valence) were faster than compatible responses (i.e., turn toward stimuli of positive valence). They suggested that this reverse compatibility effect occurred because position-based activation involves a participant's intentions and goals. Thus, the observed effects might reflect processes other than direct motor activation (Müsseler et al., 2009).

Although several authors (for review, see Müsseler et al., 2009; van Peer, Rotteveel, Spinhoven, Tollenaar, \& Roelofs, 2010) proposed that positive-toward/ negative-away coding is a type of compatible condition and positive-away/negative-toward coding is an incompatible condition, no studies of which we are aware have employed simply left and right locations of stimuli and keys to investigate the effect of affective valence on spatial compatibility effects.

The main objective of the present study was to investigate whether the affective valence of stimuli influences the spatial Stimulus-Response Compatibility effect along the horizontal dimension. Classically, spatial compatibility effects along the horizontal (leftright) dimension have been considered to result from the structural organization of the nervous system, which facilitates an ipsilateral response compared with a contralateral response. According to this view (i.e., the null hypothesis), the affective valence of a stimulus will have a null or weak influence on spatial compatibility effects. In contrast to this view, the compatible response may be processed as an approach response, and the incompatible response may be processed as a withdrawal response. To discriminate between these hypotheses, we used lateralized figures of soccer players from the participant's Favorite team and its main Rival team as the positive and negative valence stimulus, respectively. We found that the affective valence of the stimulus elicits a "normal" Spatial Compatibility effect for the Favorite team and "reversed" Spatial Compatibility effect for the Rival team. These results may be explained by the approach/avoidance hypothesis and suggest that compatible and incompatible responses along the horizontal dimension may be equivalent to approach/ avoidance reactions along the near/far (Markman \& Brendl, 2005; Proctor \& Zhang, 2010) and lower/upper (Proctor \& Zhang, 2010) dimensions.

\section{Methods}

\section{Participants}

The experiment involved 14 students from the Universidade Federal Fluminense (Rio de Janeiro, Brazil), aged 19-30 years (eight male and six female). All of the participants were right-handed (Oldfield, 1971), had normal or corrected-to-normal vision, 
and were naive about the purposes of the experiment. A written informed consent form was obtained from all subjects, and the study was approved by the Institutional Ethics Commission of the Universidade Federal Fluminense (Parecer 185/2005).

\section{Valence rating}

Participants first completed a valence rating, in which they were invited to rank, in the order of preference, the names of the four main soccer teams of Rio de Janeiro (Fluminense, Flamengo, Vasco da Gama, and Botafogo). This procedure was used to select the stimuli for the Stimulus-Response Compatibility task. The first and fourth teams from the order of preference defined the Favorite and Rival soccer teams, respectively, for the participant. For one of the participants, for example, Flamengo could be the Favorite team, and Botafogo could be the Rival team. For another participant, Botafogo could be the Favorite team, and Flamengo could be the Rival team.

\section{Apparatus and stimuli}

The experiments were performed in a dimly lit and sound-proof chamber where the participants sat in front of a monitor at a viewing distance of approximately $57 \mathrm{~cm}$. Responses were made on a computer keyboard using left (the letter " $A$ ") and right (the number " 6 " on the numerical keypad) keys in the horizontal dimension. The index fingers were used to produce the experimental responses. The stimuli were realistic, full-color, threequarter profile figures of soccer players $\left(1.5^{\circ} \times 6.5^{\circ}\right.$, width and height, respectively) that represented the four popular soccer teams of Rio de Janeiro (Fig. 1). Stimuli were randomly presented at $6.0^{\circ}$ left or right from the fixation point. E-Prime software (version 2.0) was used to present the stimuli and record MRTs. Importantly, the feature that indicated a correct response was the team shirt (two among Flamengo, Botafogo, Vasco, or Fluminense), not the affective valence (Favorite/Rival).
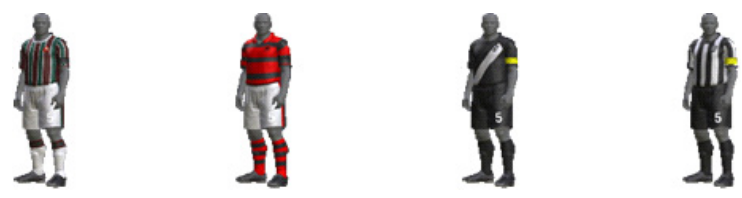

Figure 1. Figures representing the four popular soccer teams of Rio de Janeiro. (Fluminense, Flamengo, Vasco da Gama, and Botafogo).

\section{Procedure}

The participants ran two counterbalanced blocks of 120 trials each. In each experimental block, the figures of the Favorite and Rival soccer teams of the participant were used as experimental stimuli. Before each experimental block, a training block of 40 trials was run. The participants were instructed to respond by pressing the key located on the same side as the stimulus for one team (compatible condition) and by pressing the key located on the opposite side as the stimulus for the other team (incompatible condition). For half of the participants, in the first block, the compatible condition was used for the Favorite team player, and the incompatible condition was used for the Rival team player. The participant had to press the key located on the same side for the Favorite team player and the opposite key for the Rival team player (Fig. 2). In the second block, the compatibility condition of the teams was reversed. The other half of the participants began with the compatible condition for the Rival team and incompatible condition for the Favorite team. In the second block, the compatibility condition was reversed.

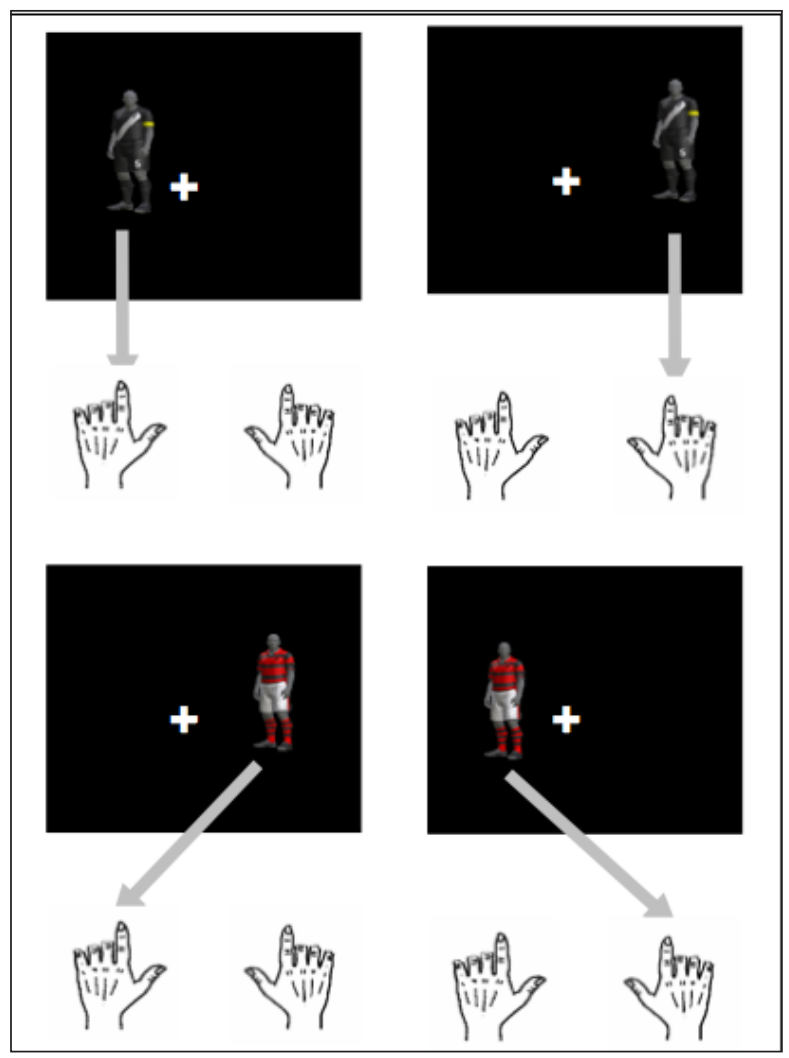

Figure 2. Compatible and incompatible experimental conditions. Participants had to press the key located on the same side as the Favorite team player (upper) and the opposite key for the Rival team player (lower). In the second block, the compatibility condition was reversed.

\section{Experimental design}

The means of the correct MRT data were subjected to analysis of variance (ANOVA) with Preference (Favorite vs. Rival), Hemifield (Left vs. Right), and Response Key (Left vs. Right) as the within-subject factors and MRT as the dependent variable. The significance level was considered $\alpha<.05$. 


\section{Results}

\section{Manual reaction time}

The main factors Preference, Hemifield, and Response Key and the two-way interactions between factors had no overall significant effects on MRT. A three-way interaction was found between Preference, Hemifield, and Response Key $\left(F_{1,13}=6.601 ; p=.023\right)$. The three-way interaction suggests that the Preference variable influenced the Hemifield-Response Key interaction. However, the Preference effect was not necessarily equal for each Hemifield or Response Key. That is, Preference may have had a stronger effect on the Right hemifield than on the Left hemifield or on the Right key than on the Left key. To ascertain the significant differences in the three-way interaction (Fig. 3), we conducted orthogonal planned comparisons, which are more appropriate than traditional post hoc tests for investigating stimulus-response compatibility tasks (Keppel, 1991; Tagliabue et al., 2007).

The analysis of the triple interaction revealed an effect of team Preference (Favorite or Rival) on the pattern of spatial compatibility (Fig. 3). For the Favorite team, the right key response was faster for the right stimulus (609 ms; compatible condition) than for the left stimulus

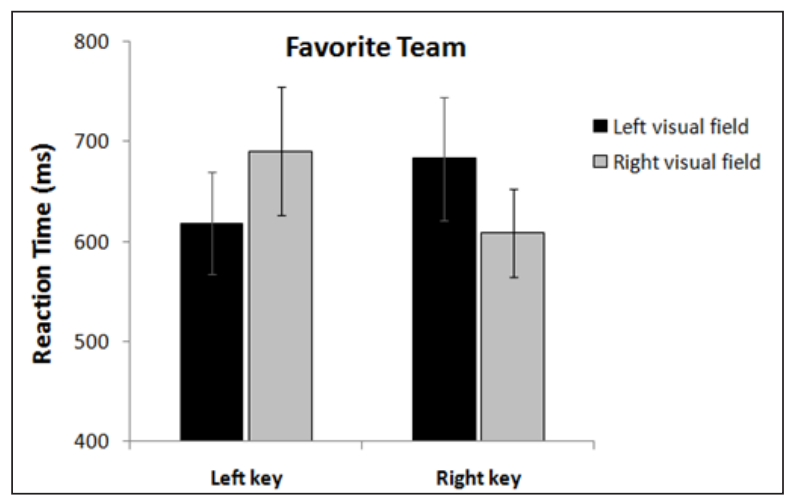

(incompatible condition; $690 \mathrm{~ms} ; p=.032$ ). A similar trend was found for the left key, but the comparison between the compatible and incompatible conditions (left stimulus-left key, 618 ms; right stimulus-left key, 683 ms) was not significant ( $p=.098$; Table 1, lower row, key correspondence effects for Favorite team). The same pattern was observed for the stimulus correspondence effect (Table 1, right column), in which the right side stimulus elicited a faster response with the right key (609 $\mathrm{ms})$ than with the left key (683 ms; $p=.037)$, and the response for the left side stimulus was faster with the left key (618 ms) than with the right key (690 ms; $p=.081)$.

Interestingly, for the Rival team, an opposite pattern of spatial compatibility was found, and the participants responded faster with the key located on the opposite side as the stimulus than with the key located on the same side as the stimulus. More specifically, participants responded faster with the left key for a right stimulus (603 ms; incompatible condition) than for the compatible conditions (left key-left stimulus, $698 \mathrm{~ms} ; p$ $=.012$; right key-right stimulus, $692 \mathrm{~ms} ; p=.022)$. The responses were also faster for the right key-left stimulus incompatible condition ( $626 \mathrm{~ms}$ ) than for the compatible conditions (left key-left stimulus condition, $698 \mathrm{~ms}, p=$ .017 ; right key-right stimulus, $626 \mathrm{~ms}, p=.058$ ).

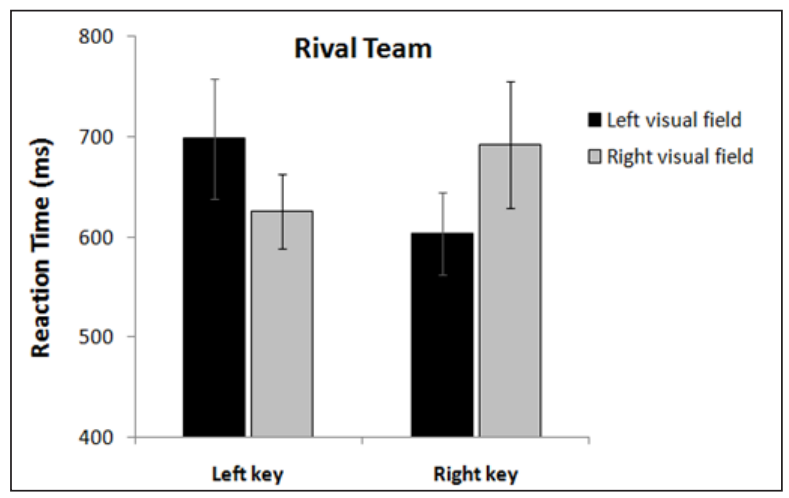

Figure 3. Mean manual reaction times for the Favorite and Rival teams presented in the left and right visual fields. Participants responded using their left and right hands (left and right key).

Table 1. Comparisons between compatible and incompatible conditions for the hemifield-response key combinations.

\begin{tabular}{|c|c|c|c|c|}
\hline \multirow{2}{*}{ Team } & \multirow{2}{*}{ Visual Field } & \multicolumn{2}{|c|}{ Response key } & \multirow{2}{*}{ Hemifield correspondence effect } \\
\hline & & Left & Right & \\
\hline \multirow[t]{2}{*}{ Favorite } & Left & 618 & 690 & $72(\mathrm{p}<.110)$ \\
\hline & Right & 683 & 609 & $74(\mathrm{p}<.039)$ \\
\hline \multicolumn{2}{|c|}{ Key correspondence effect } & $75(\mathrm{p}=.093)$ & $81(\mathrm{p}<.04)$ & \\
\hline \multirow[t]{2}{*}{ Rival } & Left & 698 & 626 & $-72(\mathrm{p}<.022)$ \\
\hline & Right & 603 & 692 & $-89(\mathrm{p}<.029)$ \\
\hline \multicolumn{2}{|c|}{ Key correspondence effect } & $-95(\mathrm{p}<.016)$ & $-66(p=.069)$ & \\
\hline
\end{tabular}


Table 2. Comparison between Favorite and Rival team MTRs for hemifield-response key combinations.

\begin{tabular}{lccccc}
\hline \multirow{2}{*}{ Team } & \multicolumn{2}{c}{ Left Visual Field } & & \multicolumn{2}{c}{ Right Visual Field } \\
\cline { 2 - 3 } \cline { 5 - 6 } & Left Key & Right Key & & Left Key & Right Key \\
\hline Favorite & 618 & 690 & & 683 & 609 \\
Rival & 698 & 626 & & 602 & 692 \\
Rival-Favorite difference & $80(\mathrm{p}<.03)$ & $-74(\mathrm{p}=.08)$ & & $-81(\mathrm{p}<.05)$ & $83(\mathrm{p}<.01)$ \\
\hline
\end{tabular}

The influence of affective valence on the spatial compatibility effect may also be evaluated by comparing the responses for the Favorite and Rival team players under each key-stimulus condition (Table 2). Although the difference in the left stimulus-right key condition was not significant $(p=.081)$, the differences in MRT for the Favorite and Rival teams in all of the other stimulus-key relationships were significant $(p<.05)$.

We can conclude that the affective valence of the stimulus may reverse the compatibility effect. For each hemifield-response combination, the modulation of the stimulus compatibility effect by its affective valence was significant or approached significance (Tables 1 and 2).

\section{Analysis of errors}

The main factors Preference, Hemifield, and Response Key had no overall significant effects on the number of correct responses. We found a significant twoway interaction between Hemifield and Response Key $\left(F_{1,13}=6.816 ; p=.021\right)$. The percentage of errors in the corresponding conditions was $12 \%$, and the percentage of errors in the noncorresponding conditions was $9 \%$. However, no significant three-way interaction was found between Preference, Hemifield, and Response Key $\left(F_{1,13}\right.$ $=.026 p=.873)$, indicating that, in contrast to MRT measures, Preference had no effect on the percentage of errors in the compatible and incompatible conditions.

\section{Discussion}

The null hypothesis was that the valence of the stimulus would not influence the spatial compatibility effect when the pictures that represented the Favorite and Rival soccer teams were used as relevant stimuli. Spatial compatibility is mainly attributable to the structural organization of the nervous system, which facilitates ipsilateral responses compared with contralateral responses. However, our results do not support this hypothesis. For the Favorite team pictures, participants responded faster using the key located on the same side as the stimulus, but the reverse pattern of responses was found for the Rival team, with participants responding faster with the key located on the opposite side as the stimulus.

These results are consistent with previous work, in which approach and withdrawal behavior were elicited by positive (agreeable) and negative (aversive) stimuli
(Chen \& Bargh, 1999; De Houwer \& Eelen, 1998; De Houwer et al., 2001; Markman \& Brendl, 2005; Proctor \& Zhang, 2010; Müsseler et al., 2009; van Peer et al., 2010; Zhang \& Proctor, 2008).

However, the experimental designs of these studies differed widely with regard to the type of relevant and irrelevant features of the stimuli, spatial locations of the stimuli and responses, and conflicts of the task (see below). One important distinction is whether the selection of the correct response is based on the affective valence of the stimulus or on another feature of the stimulus. In the former case (similar to the spatial compatibility task), the affective valence must be explicit and consciously processed before the response selection. By contrast, in the latter case (similar to the Simon classic task), the affective valence is an irrelevant feature for correct response selection, but it implicitly influences the response due to automatic facilitation or inhibition according to the congruency (or incongruency) between the affective valence of the stimulus and correct response.

In De Houwer and Eelen (1998), the affective valence of a stimulus was used as an irrelevant feature. In their experiment, positive and negative words were presented at the center of the screen, and vocal responses were recorded. Correct responses were determined according to the grammatical category (noun or adjective) of the word. Although participants were explicitly instructed to ignore the affective meanings of the presented words, verbal response times were faster when the affective valence of the presented word and correct response were the same (i.e., both were emotionally positive or negative) compared with when they differed (one positive and the other negative). These results support the hypothesis that the stimulus valence is processed automatically and facilitates or inhibits the response to the imperative feature. Similar results were obtained by De Houwer et al. (2001), in which words or pictures and verbal or nonverbal responses were used in four experiments. In Experiment 1, the names of animals or persons with positive or negative valence were presented centrally, and volunteers had to use the semantic category (animal/person) to respond verbally ("positive"/"negative"). In Experiment 2, nouns and adjectives (condition A) or words in diverse letter case (condition B) were presented, and the correct "positive" 
or "negative" verbal response was determined according to the grammatical category of the word or letter case, respectively. The "positive"/"negative" words were also used in Experiment 3, in which black and white and color pictures of positive or negative man-made objects or natural objects were presented, and the response selection was based on whether the picture was a manmade or natural object (half of participants) or if the picture was black-white or colored (the other half). Finally, in Experiment 4, positive and negative nouns and adjectives were centrally presented, and the participants were asked to move a manikin toward or away from the word, depending on its grammatical category, not on its affective valence. In short, De Houwer et al. (2001) showed that, in all of the experimental conditions, the stimulus valence was processed automatically and influenced the reaction time according to the congruency (or incongruency) between the stimulus valence and correct response. Similar experiments were performed by Zhang and Proctor (2008).

Other authors used the affective valence of the stimulus as the relevant feature for response selection. For example, Chen and Bargh (1999) used a lever that had to be pushed away (avoidance) or pulled toward (approach) the participant's body according to the affective valence of a centrally presented word. Participants were randomly assigned to one of two experimental conditions. In the incongruent condition, the participants were instructed to push the lever forward with their hand as quickly as possible when they judged the word as good and to pull the lever toward them when they judged the word as bad. In the congruent condition, participants were given the opposite instructions: pull the lever if the word is positive and push the lever if the word is negative. They found that automatic valence evaluation results in faster approach responses to positive valence stimuli and faster avoidance responses to negative valence stimuli. These results were supported by Markman and Brendl (2005) and Proctor and Zhang (2010).

The left-right dimension was only explored by Müsseler et al. (2009), in which participants had to turn a steering wheel clockwise or counterclockwise, either toward the side of a waving pedestrian to pick the person up (compatible stimulus-response relationship) or away from a careless pedestrian to avoid hitting the person (incompatible stimulus-response relationship). Incompatible responses were faster and more accurate than compatible responses, reversing the spatial compatibility effect. In contrast, when the researchers presented the pedestrians more peripherally with the intention of eliciting a less dangerous perception in the participants (Experiment 2), a spatial compatibility effect was found. In Experiment 3, participants made left-right joystick responses to a vicious dog or puppy in a walking scenario. The instructions were to avoid the vicious dog and approach the puppy or vice versa. The results showed an advantage of the spatially incompatible response and indicated that the stimulus location did not activate an automatic corresponding response and that the valence of the stimuli overruled the spatial compatibility effect in this specific traffic situation. This interpretation is consistent with results that showed that intentions, different life experiences, and even previous practice with incompatible trials can reduce or invert spatial compatibility effects (Lavender \& Hommel, 2007; Pellicano et al., 2010; Tagliabue, Zorzi, Umiltà, \& Bassignani, 2000).

The data of the present study are similar to the results of Müsseler et al. (2009), but the participants in the previous study were given clear instructions to approach or avoid the stimulus, with movements of the steering wheel (or joystick) that easily represented approach/ avoidance reactions. Our procedure, in contrast, did not mention approach or avoidance movements in the direction of the stimuli. The response was simply a key press located on the same or opposite side as the soccer team player. If we consider that the key pressed on the same side as the stimulus was an approach response to the stimulus, and the key pressed on the opposite side was an avoidance response, our results suggest that compatible and incompatible responses along the horizontal dimension may be equivalent to approach/ avoidance reactions along the near/far (Markman \& Brendl, 2005; Proctor \& Zhang, 2010) and lower/upper (Proctor \& Zhang, 2010) dimensions.

In summary, our work extends the results of previous studies in three important aspects. First, compatible and incompatible responses along the horizontal (left-right) dimension may be equivalent to approach/avoidance reactions along the near/far and lower/upper dimensions. By using affective valence stimuli, obtaining normal or reverse spatial compatibility effects is possible using a very simple experimental design, similar to a classic experiment that employed left/right stimulus and left/ right key locations (Anzola et al., 1977). Second, no difference was observed in MRT between the Favorite and Rival team players. Thus, the Preference variable influenced only the stimulus/key interaction and had no absolute influence on MRT, possibly because the arousal/ alertness effects elicited by the Favorite and Rival teams were not different. Third, antagonism between the Favorite and Rival soccer teams may reflect universal (approach/avoidance) reactions to contrasting views in relation to other sports, people, music, politicians, and so on. Thus, the affective spatial compatibility task may be a useful tool for investigating stimulus valence effects on a variety of tasks, in addition to those that involve orienting attention to a spatial position and the coding of stimulus-response spatial positions.

\section{Acknowledgements}

We wish to thank CAPES, CNPq, FAPERJ, PIBIC$\mathrm{UFF} / \mathrm{CNPq}$, and PROPP - UFF for financial support, Prof. Carlo Umiltà and Prof. Mariaelena Tagliabue 
(Università degli Study di Padova, Italy), and Prof. Lucia Riggio (Università degli Study di Parma, Italy) for their suggestions on the experimental design and statistical analysis, and Prof. John Findlay (University of Durham, UK) for his wise and very helpful criticisms and suggestions on the manuscript.

\section{References}

Alves, N.T., Fukusima, S.S., \& Aznar-Casanova, J.A. (2008). Models of brain asymmetry in the processing of emotions. Psychology and Neuroscience, 1, 63-66.

Anzola, G.P., Bertoloni, G., Buchtel, H.A., \& Rizzolatti, G. (1977). Spatial compatibility and anatomical factors in simple and choice reaction time. Neuropsychologia, 15, 295-302.

Berlucchi, G., Crea, F., Di Stefano, M., \& Tassinari, G. (1977). Influence of spatial stimulus-response compatibility on reaction time of ipsilateral and contralateral hand to lateralized light stimuli. Journal of Experimental Psychology: Human Perception and Performance, 3, 505-517.

Chen, M., \& Bargh, J.A. (1999). Consequences of automatic evaluation: immediate behavioral predispositions to approach or avoid the stimulus. Personality and Social Psychology Bulletin, $25,215-224$.

Damasio, A.R. (1994). Descartes' error: emotion, reason, and the human brain. New York: G.P. Putnam.

Damasio, A.R. (2010). Self comes to mind: constructing the conscious brain. New York: Pantheon Books.

De Houwer, J., \& Eelen, P. (1998). An affective variant of the Simon paradigm. Cognition and Emotion, 12, 45-61.

De Houwer, J., Crombez, G., Baeyens, F., \& Hermans, D. (2001). On the generality of the affective Simon effect. Cognition and Emotion, 15, 189-206.

Gawryszewski, L.G., Ferreira, F.M., Santos, M., Flavia, G., Rangel, M.L., Silva-Machado, R., Lameira, A.P., \& Pereira, A., Jr. (2008) Feixes de luz e os efeitos compatibilidade espacial e cruzamento (Light beams for the study of compatibility and crossed-hand effects). Psico (PUC-RS), 39, 391-399.

Keppel, G. (1991). Design and analysis: a researcher's handbook. 3rd edition. Englewood Cliffs, N.J.: Prentice-Hall.

Lavender, T., \& Hommel, B. (2007). Affect and action: Towards an event-coding account. Cognition and Emotion, 21, 1270-1296

Lu, C.H., \& Proctor, R.W. (1995). The influence of irrelevant location information on performance: a review of the Simon and spatial Stroop effects. Psychonomic Bulletin \& Review, 2, 174-207.
Markman, A.B., \& Brendl, C.M. (2005). Constraining theories of embodied cognition. Psychological Science, 16, 6-10.

Müsseler, J., Aschersleben, G., Arning, K., \& Proctor, R.W. (2009). Reversed effects of spatial compatibility in natural scenes. American Journal of Psychology, 122, 325-336.

Oldfield, R.C. (1971). The assessement and analysis of handedness: the Edinburg inventory. Neuropsychologia, 9, 97-113.

Pellicano, A., Iani, C., Rubichi, S., Ricciardelli, P., Borghi, A.M., \& Nicoletti, R. (2010). Real-life motor training modifies spatial performance: the advantage of being drummers. American Journal of Psychology, 123, 169-179.

Poffenberger, A.T., Jr. (1912). Reaction time to retinal stimulation, with special reference to the time lost in conduction through nerve centers. Archives of Psychology, 23, 1-73.

Proctor, R.W., \& Vu, K.P.L. (2006) Stimulus-response compatibility principles: data, theory, and application. Boca Raton, F.L.: CRC Press.

Proctor, R.W., \& Zhang, Y. (2010). "Mother nature doesn't have a bullet with your name on it": coding with reference to one's name or object location? Journal of Experimental Social Psychology, 46, 336-343.

Riggio, L., Gawryszewski, L.G., \& Umiltà, C. (1986) What is crossed in crossed-hand effects. Acta Psychologica, 62, 89-100.

Rubichi, S., \& Nicoleti, R. (2006) The Simon effect and handedness: evidence for a dominant-hand attentional bias in spatial coding. Perception \& Psychophysics, 68, 1059-1069.

Tagliabue, M., Zorzi, M., Umiltà, C., \& Bassignani, F. (2000). The role of long-term-memory and short-term-memory links in the Simon effect. Journal of Experimental Psychology: Human Perception and Performance, 26, 648-670.

Tagliabue, M., Vidotto, G., Umiltá, C., Altoè, G., Treccani, B., \& Spera, P. (2007). The measurement of left-right asymmetries in the Simon effect: a fine-grained analysis. Behavior Research Methods, 39, 50-61.

Tamietto, M., \& de Gelder, B. (2010) Neural bases of the nonconscious perception of emotional signals. Nature Reviews Neuroscience, 11, 697-709.

Umiltá, C., \& Nicoletti, R. (1990). Spatial stimulus-response compatibility. In R.W. Proctor, \& T.G. Reeve (Eds.), Stimulusresponse compatibility: an integrated perspective (pp. 89-116). Amsterdam: Elsevier.

van Peer, J.M, Rotteveel, M., Spinhoven, P., Tollenaar, M.S., \& Roelofs, K. (2010) Affect-congruent approach and withdrawal movements of happy and angry faces facilitate affective categorisation. Cognition and Emotion, 24, 863-875.

Zhang, Y., \& Proctor, R.W. (2008). Influence of intermixed emotionrelevant trials on the affective Simon effect. Experimental Psychology, $55,409-416$ 